\title{
Attraction-limited cluster-cluster aggregation of Ising dipolar particles
}

\author{
Naoki Yoshioka, ${ }^{1, *}$ Imre Varga, ${ }^{2, \dagger}$ Ferenc Kun, ${ }^{2, *}$ Satoshi Yukawa, ${ }^{1, \S}$ and Nobuyasu Ito ${ }^{1, \|}$ \\ ${ }^{1}$ Department of Applied Physics, School of Engineering, The University of Tokyo, 7-3-1, Hongo, Bunkyo-ku, Tokyo, 113-8656, Japan \\ ${ }^{2}$ Department of Theoretical Physics, University of Debrecen, P. O. Box 5, H-4010 Debrecen, Hungary
}

(Received 29 July 2005; revised manuscript received 21 October 2005; published 13 December 2005)

\begin{abstract}
The attraction-limited cluster-cluster aggregation of two-dimensional Ising dipolar particles with or without particle-size dispersity is studied. The fast decrease of the number of even-sized clusters for relatively smaller clusters is observed. The threshold concentration is also determined, under which the dynamics of aggregation is explained using the well-known dynamic scaling theory of Vicsek and Family. Above the threshold concentration, the dynamics depends on particle-size dispersity. Furthermore, it is suggested that, even in the dilute limit, the dynamic exponents are affected by the screening of the surrounding clusters on collision between two clusters.
\end{abstract}

DOI: 10.1103/PhysRevE.72.061403

PACS number(s): 61.43.Hv, 45.50.-j, 82.70.Dd, 83.10.Pp

\section{INTRODUCTION}

The structure formation in a monolayer of dipolar particles has been a subject of academic and practical interest for several decades for the following reasons. Dipolar particles have long-ranged anisotropic dipole-dipole interaction, and they are readily controllable using external fields. In the absence of external fields, they form randomly oriented chains, closed rings, and fractal aggregates [1-3]. In an external field, in contrast, they form chains in the direction of the field $[2,4-6]$, or a crystal structure emerges if the angle of the field with a surface tangent is larger than a threshold angle $[7,8]$.

Cluster-cluster aggregation (CCA) in such a system becomes more complicated than that of particles that experience shorter-ranged interactions [2-6]. For the latter case, sticking and breakup probabilities between clusters play essential roles, and several models like diffusion-limited CCA (DLCA) are known [9]. In contrast, for the former case, the geometric and dynamic features of CCA depend on details of the interactions, e.g., the magnitude of dipole moments and that of an external field. If the contribution of these quantities to CCA is as large as or larger than that of thermal diffusion, it appears that the behavior of CCA deviates from the predictions using simple models, such as DLCA. Although there have been several studies of CCA of dipolar particles, the comprehension of CCA of dipolar particles is far from complete.

Recently, Varga et al. have proposed unusual system of a monolayer of dipolar particles [10]. In their system, the particles are confined to a plane and have Ising dipole moments, i.e., their dipole moments are constrained to be perpendicular to the plane. Thus, we call them Ising dipolar particles (IDPs). IDPs are classified into two components depending

\footnotetext{
*Electronic address: yoshioka@acolyte.t.u-tokyo.ac.jp

†Electronic address: vargai@dtp.atomki.hu

"Electronic address: feri@dtp.atomki.hu

${ }^{\S}$ Electronic address: yukawa@ap.t.u-tokyo.ac.jp

"Electronic address: ito@ap.t.u-tokyo.ac.jp
}

on whether their dipole moments point upward or downward. These components may also differ in other physical properties, e.g., the magnitude of dipole moments and the size of particles. IDPs in a plane are expected to be a simple and relatively natural model because they experience isotropic interaction and can be regarded as oppositely charged particles. Since these charges frustrate the particles, they show rich varieties of structure formation [10].

To date, two physical realizations of IDPs in a plane have been proposed: a deposited mixture of silica and polystyrene colloids subjected to an ac electric field in an electromagnetically passive liquid [11]; and macroscopic particles with permanent magnetic dipole moments on an airwater interface [12]. The former is, however, not simply regarded as a system of IDPs in a plane; electrohydrodynamic flow [13] and thermal diffusion affect the structure formation of the colloids. In contrast, the latter is an appropriate example and gives qualitative agreement with the results of the simulations $[10,12]$.

In this paper, we examine the dynamic features of the CCA of IDPs experimentally and numerically; this is a simple and realistic model of attraction-limited CCA (ALCA) of dipolar particles, or a limiting case in which thermal diffusion is ignored. ALCA has been given little importance, except for systems of electrorheological (ER) suspensions in a strong electric field [4]. However, they do not satisfy us because of the anisotropy of dipole-dipole interactions, which is different from the characteristics of the IDPs described above. The CCA of IDPs is also interesting as ALCA of oppositely charged particles, in connection with the heteroaggregation of oppositely charged colloids $[14,15]$.

We find the fast decrease of the number of even-sized clusters for relatively smaller clusters. We also obtain the average cluster size and the total number of clusters as functions of time. They obey the power-law behavior and their dynamic exponents are equal if the concentration of IDPs is lower than the threshold. It is considered that the dynamic scaling theory [16] is valid within the concentration range. Above the threshold, the dependence of their dynamics on particle-size dispersity is found. Furthermore, we consider the physical origin of the dynamic exponents in the dilute limit. 




(a)

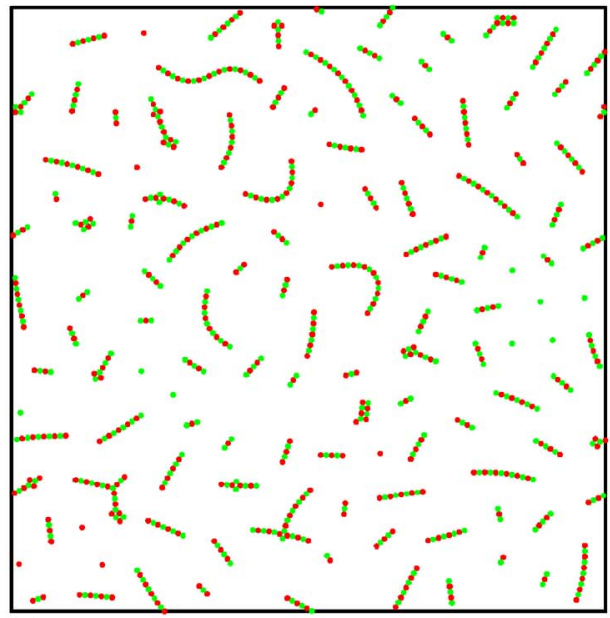

(c)

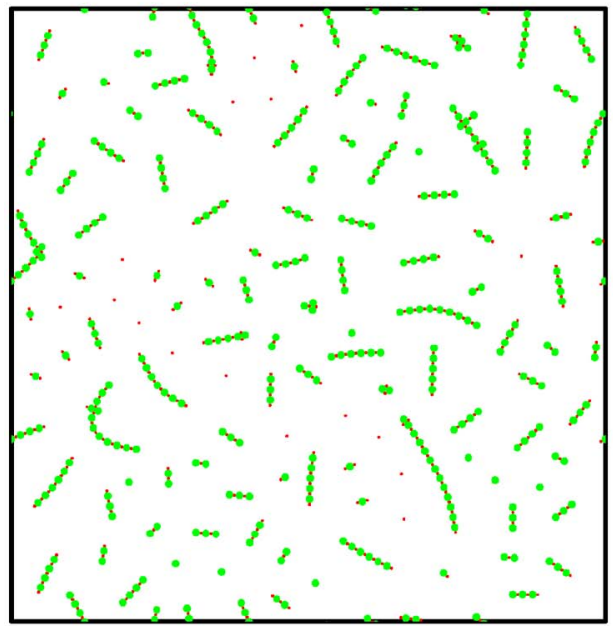

(e)

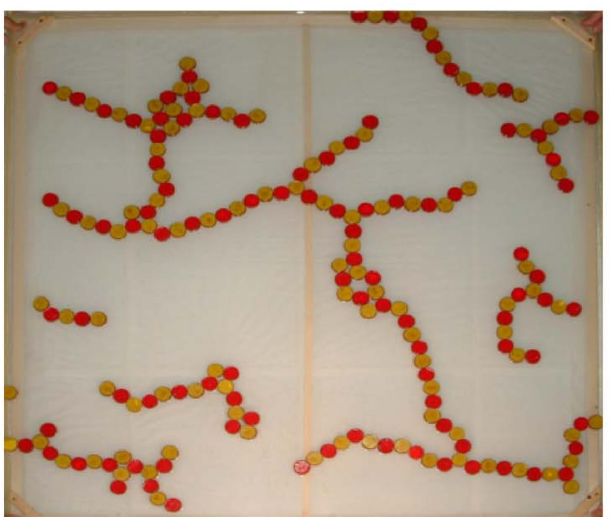

(b)

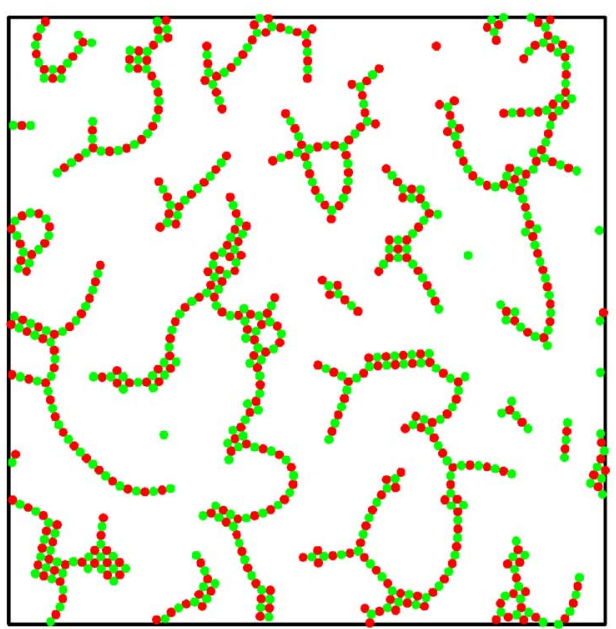

(d)

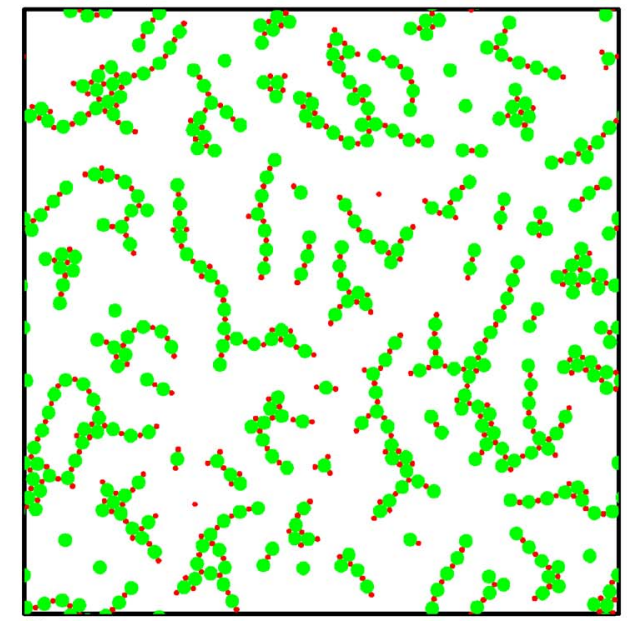

(f)
FIG. 1. (Color online) Snapshots of typical configuration in experiment [(a) and (b)] and simulation [(c)-(f)]: (a) $\phi=0.075$, $\sigma=1.0$; (b) $\phi=0.105, \sigma=1.0$; (c) $\phi=0.05, \quad \sigma=1.0 ; \quad$ (d) $\phi=0.125$, $\sigma=1.0$; (e) $\phi=0.05, \sigma=2.6$; (f) $\phi=0.175, \sigma=2.6$. Red (dark gray) disks are particles with upward dipole moments, and yellow or green (light gray) disks are those with downward dipole moments.

\section{METHODS}

\section{A. Experimental setup}

We constructed an experimental technique that enables a straightforward and controllable realization of binary monolayers with particles of oppositely oriented dipole moments constrained to be perpendicular to the plane of motion. In the experimental setup, macroscopic particles are constructed by attaching magnetized cylindrical metal particles to floats. The floats are cork disks that ensure the confinement of com- posite particles to the air-water interface (floating) and prevent flipping, constraining the dipole moment of the particles perpendicular to the water surface. The two components of the system are realized by the two opposite orientations of the dipole moments of the particles. To ensure that all the particles are released at the same time from the initial position, a hard plastic net was fixed on a wooden frame, which can float on the surface of water. The initial state is prepared such that a square container of side length $L=500 \mathrm{~mm}$ is filled with water with a plastic net floating on the surface. 


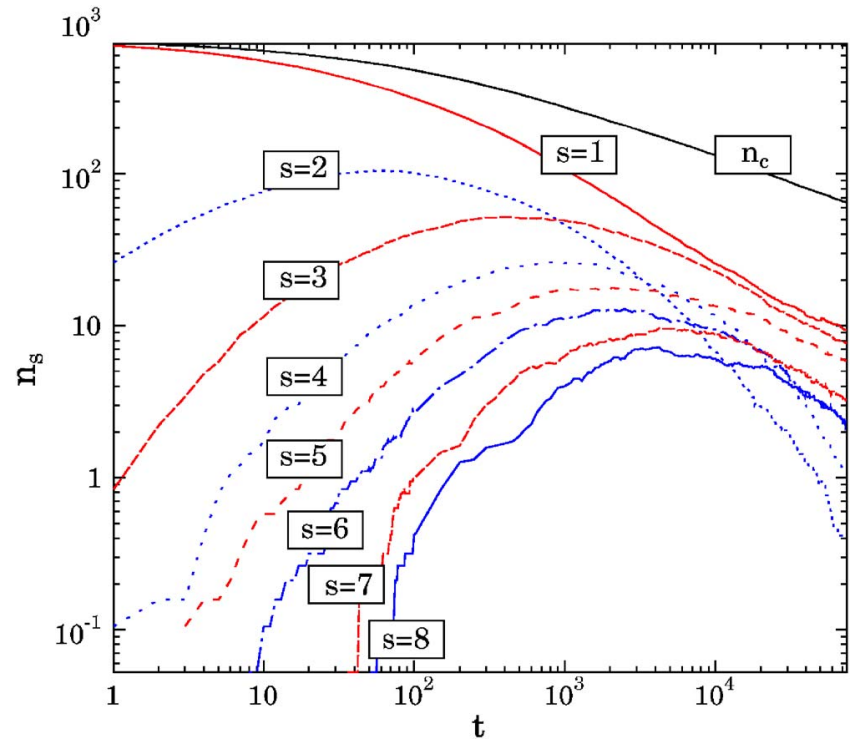

FIG. 2. (Color online) Time evolution of the numbers of $s$-clusters for $\phi=0.1, \sigma=1.0$. The thick solid line denotes the total number of clusters.

The particles are placed randomly onto the net which keeps them fixed in their initial position. By immersing the net into the container, the particles start to move at the same time resulting in a cluster-cluster aggregation. In all the experiments, the particle radius $R$ and the magnitude of dipole moments $\mu$ were the same for the two components. The concentration of the system, $\phi=N \pi R^{2} / L^{2}$, was controlled by varying the number of particles $N$, while the size of the container was fixed.

Experiments were carried out at three different concentrations $\phi=0.05,0.075$, and 0.105 repeating the experiments ten times with different random initial states. The time evolution of the aggregation was recorded using a video camera. Quantities characterizing the dynamics of aggregation were determined from the digital image, averaging over independent realizations. Figures 1(a) and 1(b) show typical snapshots of the experiments.

\section{B. Numerical simulation}

In contrast, we construct a numerical model to understand the behavior of the particles shown in the experiment. We treat an ensemble $\Pi_{N}=\{i \in \mathbb{N} \mid 1 \leqslant i \leqslant N\}$ of $N=750$ particles in a periodic square box of side length $L$. Each particle has a pointlike dipole moment in its center, and the dipole moment is fixed to be perpendicular to the plane of motion during the time evolution of the system. There are two types of particles in this system: type + consists of $N_{+}$particles with radius $R_{+}$and dipole moment $\mu_{+}>0$ pointing upward, and type - consists of $N_{-}=N-N_{+}$particles with radius $R_{-}$ and dipole moment $\mu_{-}<0$ pointing downward. We consider only three forces that a particle $i \in \Pi_{N}$ experiences: the dipolar force

$$
\boldsymbol{F}_{i j}^{\mathrm{dd}}=-\frac{3 \mu_{i} \mu_{j}}{r_{i j}^{4}} \boldsymbol{n}_{i j}
$$

the Stokes force

$$
\boldsymbol{F}_{i}^{\mathrm{hyd}}=-\alpha_{i} \frac{d \boldsymbol{r}_{i}}{d t},
$$

and the Hertz contact force

$$
\boldsymbol{F}_{i j}^{\mathrm{pp}}=-k_{i j}^{\mathrm{pp}} h_{i j} H\left(h_{i j}\right) \boldsymbol{n}_{i j} ;
$$

$j \in \Pi_{N} \backslash\{i\}$ is another particle, $\boldsymbol{r}_{i}$ is the position vector of the center of $i, \mu_{i}=\mu_{+}$or $\mu_{-}$is the dipole moment of $i, R_{i}$ is the radius of $i, r_{i j}=\left|\boldsymbol{r}_{j}-\boldsymbol{r}_{i}\right|, \boldsymbol{n}_{i j}=\left(\boldsymbol{r}_{j}-\boldsymbol{r}_{i}\right) / r_{i j}, h_{i j}=R_{i}+R_{j}-r_{i j}$, and $H(x)$ is the Heaviside step function.

The system is supposed to be fully dissipative; hence the equation of motion of this system is written as

$$
\sum_{j \in \Pi_{N} \backslash\{i\}}\left(\boldsymbol{F}_{i j}^{\mathrm{dd}}+\boldsymbol{F}_{i j}^{\mathrm{pp}}\right)+\boldsymbol{F}_{i}^{\mathrm{hyd}}=\mathbf{0}
$$

for any $i \in \Pi_{N}$. It is not necessary to set the coefficients $\left\{\alpha_{i}\right\}$ and $\left\{k_{i j}^{\mathrm{pp}}\right\}$ in detail so that we take $\alpha_{i}$ to be unity for any $i$ and choose $k_{i j}^{\mathrm{pp}}$ as a constant $k^{\mathrm{pp}}$ independent of $i$ and $j$. Therefore, the equation of motion is rewritten as

$$
\frac{d \boldsymbol{r}_{i}}{d t}=-\sum_{j \in \Pi_{N} \backslash\{i\}}\left(\frac{3 \mu_{i} \mu_{j}}{r_{i j}^{4}}+k^{\mathrm{pp}} h_{i j}^{3 / 2} H\left(h_{i j}\right)\right) \boldsymbol{n}_{i j} .
$$

We perform molecular dynamics (MD) simulations, i.e., the time evolution of the system is followed by solving Eq. (1) numerically, using the adaptive fourth-order Runge-Kutta scheme. To ensure the disorder of the initial configuration, pointlike particles without dipole moments are first placed in the simulation box randomly and independently. Then the particles are gradually enlarged, i.e., their radii are gradually increased such that after each increment, MD simulation is performed taking into account the repulsive force arising between overlapping particles. As a result of the simulation, all the particles can find an equilibrium, overlap-free position. This procedure is repeated until the radius of each particle $i$ reaches the desired $R_{i}$. After these initialization procedures, we perform the MD simulation of the particles with dipole moments. For each particle $i$, the dipolar forces $\boldsymbol{F}_{i j}^{\mathrm{dd}}$ are summed up for the particles $\left\{j \in \Pi_{N} \backslash\{i\} \mid \boldsymbol{r}_{i}=\left(x_{i}, y_{i}\right)\right.$ such that $\left.\boldsymbol{r}_{j} \in\left[x_{i}-L / 2, x_{i}+L / 2\right) \times\left[y_{i}-L / 2, y_{i}+L / 2\right)\right\}$. Thus, the cutoff length of the dipolar forces is approximately $L / 2$.

Simulations have been carried out fixing $\phi_{\mathrm{r}}=N_{-} / N_{+}=1.0$ and $\mu_{\mathrm{r}}=\left|\mu_{-} / \mu_{+}\right|=1.0$, and varying the concentration $\phi=\left(N_{+} \pi R_{+}{ }^{2}+N_{-} \pi R_{-}^{2}\right) / L^{2}$. In addition, we set the relative particle radius $\sigma=R_{-} / R_{+}$to 1.0 and 2.6. The latter means that a smaller (type + ) particle cannot touch four larger (type -) particles at the same time. Figures 1(c)-1(f) show typical snapshots of the simulations.

\section{RESULTS}

\section{A. Cluster discrimination}

We observe behavior peculiar to binary systems, which was not observed in previous studies of CCA of dipolar particles. Figure 2 shows a typical graph of the number of clusters $n_{s}(t) L^{2}$ for each small $s$ as a function of time $t ; n_{s}(t)$ is the dynamic cluster size distribution, or the number of clusters involving $s$ particles ( $s$-clusters) per unit area at time $t$. It 

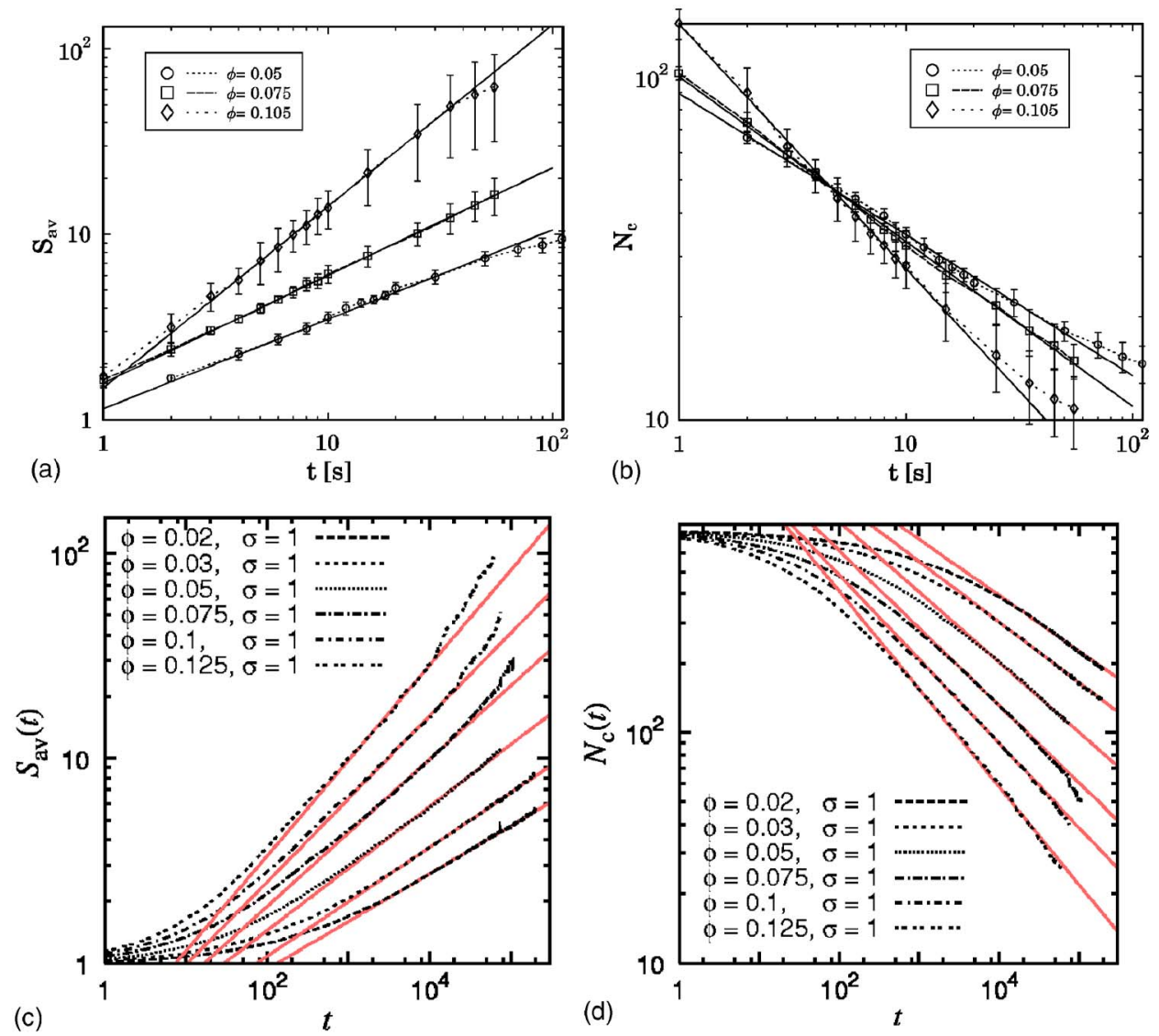

FIG. 3. (Color online) Log-log plots of the average cluster size $[(\mathrm{a}),(\mathrm{c})$, and (e)] and total number of clusters [(b), (d), and (f)] as functions of time. (a) and (b) are the result of the experiment at $\sigma=1.0$, (c) and (d) that of simulation at $\sigma=1.0$, and (e) and (f) that of simulation at $\sigma=2.6$.

(d)
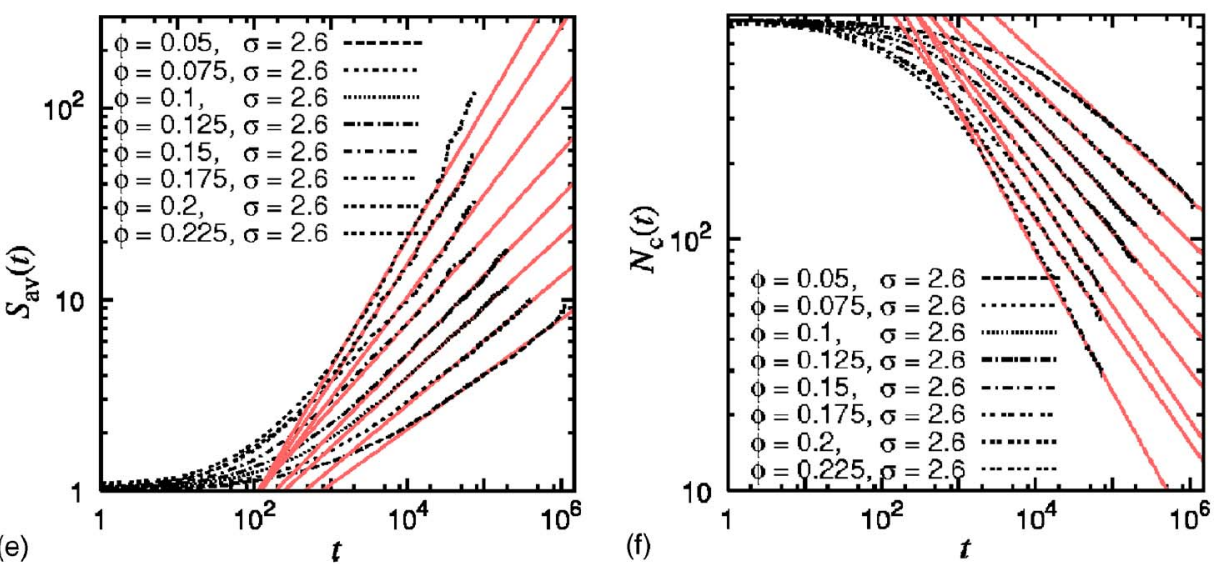

(f)

is clearly observed that, for relatively smaller $s$ 's such as $s=1,2,3$, and 4 , the number of even-sized clusters decreases faster than that of odd-sized ones. That is, the number of neutral clusters decays faster than that of charged ones. This behavior is known as cluster discrimination in CCA of oppositely charged colloids $[14,15]$. Since smaller clusters have chainlike structures [see Figs. 1(a), 1(c), and 1(e)], they can join other clusters only at chain ends. Odd-sized clusters, however, can form aggregates only with clusters that have oppositely charged particles, at least, at one of their ends because they have equally charged particles at both ends. In contrast, even-sized clusters have differently charged particles at both ends. Therefore, the reactivities of even-sized clusters are higher than those of odd-sized ones.

\section{B. Dynamic exponents}

Figures 3(a)-3(f) show the average cluster size

$$
S_{\mathrm{av}}(t)=\frac{\sum_{s} s^{2} n_{s}(t)}{\sum_{s} s n_{s}(t)}
$$

and the total number of clusters

$$
N_{\mathrm{c}}(t)=\sum_{s} n_{s}(t) L^{2}
$$

for each concentration as functions of time $t$. It appears that the curves have a time range that obeys the power-law be- 
TABLE I. Dynamic exponents $z$ and $z^{\prime}$.

\begin{tabular}{|c|c|c|c|c|c|c|}
\hline \multirow[b]{3}{*}{$\phi$} & \multirow{2}{*}{\multicolumn{2}{|c|}{$\begin{array}{c}\text { Experiment } \\
\sigma=1\end{array}$}} & \multicolumn{4}{|c|}{ Simulation } \\
\hline & & & \multicolumn{2}{|c|}{$\sigma=1$} & \multicolumn{2}{|c|}{$\sigma=2.6$} \\
\hline & $z$ & $z^{\prime}$ & $z$ & $z^{\prime}$ & $z$ & $z^{\prime}$ \\
\hline 0.02 & & & 0.23 & 0.24 & & \\
\hline 0.03 & & & 0.27 & 0.26 & & \\
\hline 0.05 & 0.48 & 0.41 & 0.31 & 0.31 & 0.29 & 0.29 \\
\hline 0.075 & 0.58 & 0.48 & 0.36 & 0.34 & 0.34 & 0.30 \\
\hline 0.1 & & & 0.39 & 0.37 & 0.37 & 0.33 \\
\hline 0.105 & 0.97 & 0.71 & & & & \\
\hline 0.125 & & & 0.46 & 0.42 & 0.41 & 0.36 \\
\hline 0.15 & & & & & 0.45 & 0.40 \\
\hline 0.175 & & & & & 0.53 & 0.45 \\
\hline 0.2 & & & & & 0.62 & 0.44 \\
\hline 0.225 & & & & & 0.68 & 0.56 \\
\hline
\end{tabular}

havior: $S_{\mathrm{av}}(t) \sim t^{z}$ and $N_{\mathrm{c}}(t) \sim t^{-z^{\prime}}$. In this range, however, the curves slightly oscillate because of cluster discrimination; the curves go upward more gently when one type of evensized clusters is disappearing, and they become steeper when it has disappeared. Nevertheless, we can estimate the dynamic exponents $z$ and $z^{\prime}$. Table I and Fig. 4 show the dynamic exponents $z$ and $z^{\prime}$ as functions of concentration $\phi$.

To interpret these results, we consider the dynamic scaling theory first proposed by Vicsek and Family for DLCA [16]. They assume that the dynamic cluster size distribution is written as

$$
n_{s}(t) \sim s^{-2} f\left(\frac{s}{s^{*}(t)}\right) \sim t^{-w} s^{-\tau} g\left(\frac{s}{s^{*}(t)}\right),
$$

where $s^{*}(t)$ is the typical cluster size of CCA, and $f(x)$ and $g(x)$ are scaling functions. According to the theory, if $\tau<1$ then $S_{\mathrm{av}}(t) \sim s^{*}(t)$ and $N_{\mathrm{c}}(t) \sim\left[s^{*}(t)\right]^{-1}$, and thus $z=z^{\prime}$ is derived. In the dilute limit, it is expected that various types of CCA are described by the Smoluchowski equation, and the dynamic scaling theory can be derived using that equation [17].

In Fig. 4, the statistical errors of the dynamic exponents $z$ and $z^{\prime}$ are rather small for the size of the symbols, and their systematic errors are on the order of the deviation of the curves. Therefore, we find that $z$ and $z^{\prime}$ tend to be equal at $\phi \leqq 0.05$, while there is a discrepancy between the exponents at $\phi>0.05$. Because we verify that $\tau<1$ within the concentration range shown in Fig. 4, it is shown that there is a threshold concentration $\phi_{\mathrm{c}}=0.05$ above which the conventional dynamic scaling theory does not apply. Furthermore, we find that the exponents seem to be independent of particle-size dispersity $\sigma$ at $\phi=\phi_{\text {c }}$ while they depend on $\sigma$ at $\phi>\phi_{\mathrm{c}}$. This means that the dynamics of IDPs does not depend on $\sigma$ if the dynamic scaling theory is valid.

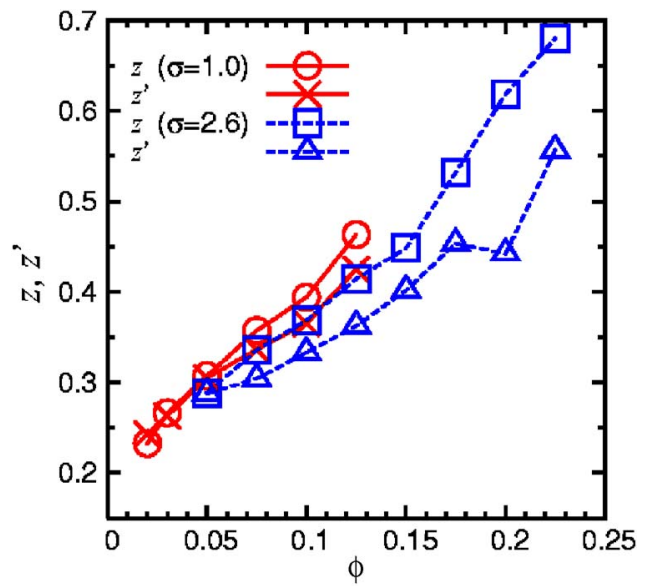

FIG. 4. (Color online) Dependence of dynamic exponents $z$ and $z^{\prime}$ on concentration $\phi$ for $\sigma=1.0$ (solid lines) and 2.6 (dashed lines) obtained from simulations. The error bars are omitted because the statistical errors of the exponents are small enough for the size of the error bars to fall within the order of the size of the symbols.

\section{Dynamic exponents in dilute limit}

In Fig. 4, it appears that the dynamic exponents become close to $0.2-0.25$ in the dilute limit. This value is much smaller than that of other CCAs, particularly that of ALCA of ER suspensions in a strong electric field [4]. This means that the dynamics of IDPs cannot be described using a hierarchical model [18] approach similar to that carried out by See and Doi [4], in which only the two-body collision between nearest neighbor $2^{k}$-clusters is considered at the $k$ th step. In fact, because the typical cluster size is of the same order as $\phi^{-1}$ when the CCA of chains crosses that of fractal aggregates [10], we can consider that all clusters are straight chains if $\phi \lesssim 1 / N$. The potential energy between two straight IDP chains of the same size $s=2^{k}$ and of the center-of-mass distance $r$ is expressed as

$$
U(r)=\mu^{2} \sum_{n=1}^{s} \sum_{m=1}^{s} \frac{(-1)^{n+m}}{\left[d_{n m}(r)\right]^{3}},
$$

where $d_{n m}(r)$ is the distance between the $n$th IDP of one chain and the $m$ th IDP of the other. If $r \gg s\left(R_{+}+R_{-}\right)$then

$$
U(r) \sim \frac{\left[\mu s\left(R_{+}+R_{-}\right)\right]^{2}}{r^{5}},
$$

and thus $z=2 / 5$ is derived using the theory of See and Doi [4]. This value deviates from what we obtain, $z(\phi \rightarrow 0)$ $=0.2-0.25$. This means that, in the case of the CCA of IDPs, the surrounding clusters and their screening effect are not negligible.

\section{SUMMARY}

In summary, we have proposed the CCA of IDPs as a model of ALCA, and have studied its dynamic features experimentally and numerically. We have found that the reactivities of even-sized clusters are higher than those of 
odd-sized ones. We have also found that the average cluster size and total number of clusters obey power-law behavior, and that the dynamic scaling theory explains the behavior of these quantities within the concentration range $\phi \lesssim \phi_{\mathrm{c}}=0.05$. Particle-size dispersity affects the behavior only at $\phi>\phi_{\mathrm{c}}$. In the dilute limit, the dynamic exponents become close to $0.2-0.25$. This is because the screening of the surrounding clusters slows down the collisions of clusters.

\section{ACKNOWLEDGMENTS}

F.K. is grateful to the Japan Society for the Promotion of Science for generous support during a research stay in Japan. This work was partly supported by JSPS Grants-in-Aid No. 14080204 and No. 14740229. F.K. and I.V. were supported by OTKA Grant No. T049209 and Hungarian-Chinese Intergovernmental Project No. CHN-14/04.
[1] W. Wen, F. Kun, K. F. Pál, D. W. Zheng, and K. N. Tu, Phys. Rev. E 59, R4758 (1999).

[2] H. Morimoto and T. Maekawa, J. Phys. A 33, 247 (2000).

[3] L. Kalachev, H. Morimoto, and T. Maekawa, Int. J. Mod. Phys. B 15, 774 (2001).

[4] H. See and M. Doi, J. Phys. Soc. Jpn. 60, 2778 (1991).

[5] M. Carmen Miguel and R. Pastor-Satorras, Phys. Rev. E 59, 826 (1999).

[6] J. Černák, G. Helgesen, and A. T. Skjeltorp, Phys. Rev. E 70, 031504 (2004).

[7] W. Wen, L. Zhang, and P. Sheng, Phys. Rev. Lett. 85, 5464 (2000).

[8] V. A. Froltsov, R. Blaak, C. N. Likos, and H. Löwen, Phys. Rev. E 68, 061406 (2003).

[9] T. Vicsek, Fractal Growth Phenomena (World Scientific, Singapore, 1989), Chap. 8.
[10] I. Varga, F. Kun, and K. F. Pál, Phys. Rev. E 69, 030501(R) (2004).

[11] W. D. Ristenpart, I. A. Aksay, and D. A. Saville, Phys. Rev. Lett. 90, 128303 (2003).

[12] I. Varga, H. Yamada, F. Kun, H.-G. Matuttis, and N. Ito, Phys. Rev. E 71, 051405 (2005).

[13] M. Trau, D. A. Saville, and I. A. Aksay, Langmuir 13, 6375 (1997).

[14] A. M. Puertas, A. Fernández-Barbero, and F. J. de las Nieves, Physica A 304, 340 (2002).

[15] J. M. López-López, A. Schmitt, J. Callejas-Fernández, and R. Hidalgo-Álvarez, Phys. Rev. E 69, 011404 (2004).

[16] T. Vicsek and F. Family, Phys. Rev. Lett. 52, 1669 (1984).

[17] F. Leyvraz, Phys. Rep. 383, 95 (2003).

[18] R. Botet, R. Jullien, and M. Kolb, J. Phys. A 17, L75 (1984). 